\title{
Noncontextual wirings
}

\author{
Barbara Amaral, ${ }^{1,2}$ Adán Cabello, ${ }^{3}$ Marcelo Terra Cunha, ${ }^{4}$ and Leandro Aolita ${ }^{5,6}$ \\ ${ }^{1}$ Departamento de Matemática, Universidade Federal de Ouro Preto, Ouro Preto, MG, Brazil \\ ${ }^{2}$ Departamento de Física e Matemática, CAP - Universidade Federal de São João del-Rei, 36.420-000, Ouro Branco, MG, Brazil \\ ${ }^{3}$ Departamento de Física Aplicada II, Universidad de Sevilla, E-41012 Sevilla, Spain \\ ${ }^{4}$ Departamento de Matemática Aplicada, IMECC-Unicamp, 13084-970, Campinas, São Paulo, Brazil \\ ${ }^{5}$ Instituto de Física, Universidade Federal do Rio de Janeiro, \\ Caixa Postal 68528, Rio de Janeiro, RJ 21941-972, Brazil \\ ${ }^{6}$ International Institute of Physics, Federal University of Rio Grande do Norte, 59070-405 Natal, Brazil
}

(Dated: May 24, 2017)

\begin{abstract}
Contextuality is a fundamental feature of quantum theory and is necessary for quantum computation and communication. Serious steps have therefore been taken towards a formal framework for contextuality as an operational resource. However, the most important component for a resource theory -a concrete, explicit form for the free operations of contextuality- was still missing. Here we provide such a component by introducing noncontextual wirings: a physically-motivated class of contextuality-free operations with a friendly parametrization. We characterize them completely for the general case of black-box measurement devices with arbitrarily many inputs and outputs. As applications, we show that the relative entropy of contextuality is a contextuality monotone and that maximally contextual boxes that serve as contextuality bits exist for a broad class of scenarios. Our results complete a unified resource-theoretic framework for contextuality and Bell nonlocality.
\end{abstract}

PACS numbers: 03.65.Ta, 03.65.Ud, 02.10.Ox

Introduction. Quantum contextuality refers to the impossibility of explaining the statistical predictions of quantum theory in terms of models where the measurement outcomes reveal pre-existent system properties whose values are independent on the context, i.e., on which (or whether) other compatible measurements are jointly performed $[1,2]$. Contextuality can be seen as a generalization of Bell nonlocality [3] to single systems, i.e., without the space-like separation restriction. It thus represents an exotic, intrinsically quantum phenomenon with both fundamental and practical implications. Contextuality has received lots of attention over the last decade. On the one hand, it has been experimentally studied in a variety of physical setups [4-8]. On the other one, it has been formally identified as a necessary ingredient for universal quantum computing [9-11] and a resource for random number certification [12], as well as for several other information-processing tasks in the specific case of space-like separated measurements [13].

This has motivated considerable interest in resource theories of both contextuality [14, 15] and Bell nonlocality [16-18]. Resource theories give powerful frameworks for the formal treatment of a physical property as an operational resource, adequate for its characterization, quantification, and manipulation $[19,20]$. Their central component is a special class of transformations, called the free operations, that fulfill the essential requirement of mapping every free (i.e., resourceless) object of the theory into a free object. Whereas resource-theoretic approaches for quantum nonlocality are highly developed $[16-18,21-25]$, the operational framework of contextuality as a resource is still incomplete. In Refs. [14, 15], an abstract characterization of the axiomatic structure of a resource theory of contextuality was done. However, a concrete specification of the free operations of contextuality was not given. Without an explicit parametrization of a physically-motivated class of free operations, a resource theory significantly loses applicability. For instance, in Refs. $[14,15]$, an interesting measure of contextuality, called the relative entropy of contextuality, was proposed, but only partial monotonicity under a rather restricted subset of contextuality free operations was shown. Monotonicity (non-increase under the corresponding free operations) is the fundamental requirement for a function to be a valid quantifier of a resource.

Here, we fill this gap by introducing the class of noncontextual wirings. These are the natural noncontextuality preserving physical operations at hand in the deviceindependent scenario of black-box measurement devices, where one does not assume any a-priori knowledge of the state or the observables in question. We derive a friendly analytical expression for generic noncontextual wirings applicable to all nondisturbing boxes, so that both quantum and post-quantum boxes are covered. In addition, the framework is versatile in that it allows for transformations between systems with different numbers of inputs and outputs as well as different compatibility constraints. Furthermore, for space-like separated measurements, the wirings reduce to local operations assisted by shared randomness, the canonical free operations of Bell nonlocality [16-18]. Hence, the framework constitutes a unified resource theory for both contextuality and Bell nonlocality in their most general forms. As applications, first, we show that an important quantifier called relative entropy of contextuality is monotonous under all noncontextual wirings, thus closing a major open problem $[14,15]$. Then, for the broad class of so-called cycle boxes, we show that contextality bits exists in the strongest possible 
sense: single boxes from which the entire nondisturbing set can be freely obtained with noncontextual wirings.

Nondisturbing boxes. We consider a black-box measurement device with $b$ buttons (inputs) and $l$ lights (outputs), with $b, l \in \mathbb{N}$, such that every time a button is pressed a light is turned on. We assume that the number of lights on is always equal to the number of buttons pressed. Not all buttons are compatible, i.e., can be pressed jointly. Each subset of compatible buttons defines a context. Let $\mathcal{X}=\{1,2, \ldots, b\}$ represent the set of buttons. The contexts can be encoded in an input compatibility hyper-graph $\mathcal{I}_{\mathcal{X}}:=\left\{\boldsymbol{x}^{(j)} \in\{0,1\}^{b}\right\}_{j=1, \ldots,\left|\mathcal{I}_{\mathcal{X}}\right|}$, where $\left|\mathcal{I}_{\mathcal{X}}\right|$ is the number of contexts and $j$ labels each context. For each $1 \leq j \leq\left|\mathcal{I}_{\mathcal{X}}\right|, x_{i}^{(j)}=0$ stands for " $i$-th button not pressed for context $j$ " and $x_{i}^{(j)}=1$ for " $i$-th button pressed for context $j "$ ". For any two strings $\boldsymbol{x}^{(j)}, \boldsymbol{x}^{\left(j^{\prime}\right)} \in \mathcal{I}_{\mathcal{X}}$, we denote by $\boldsymbol{x}^{(j)} \succeq \boldsymbol{x}^{\left(j^{\prime}\right)}$ the relationship " $x_{i}^{(j)}=0$ implies $x_{i}^{\left(j^{\prime}\right)}=0$, for all $i \in \mathcal{X}$ ". In other words, $\boldsymbol{x}^{(j)} \succeq \boldsymbol{x}^{\left(j^{\prime}\right)}$ means that all the buttons not pressed in $j$ are also not pressed in $j^{\prime}$ [so that pressing additional buttons from $\boldsymbol{x}^{\left(j^{\prime}\right)}$ can lead to $\left.\boldsymbol{x}^{(j)}\right]$. This defines a partial ordering $\mathcal{I}_{\mathcal{X}}$, and we say that $j$ is a maximal context if, for all $1 \leq j^{\prime} \leq\left|\mathcal{I}_{\mathcal{X}}\right|, \boldsymbol{x}^{\left(j^{\prime}\right)} \succeq \boldsymbol{x}^{(j)}$ implies $\boldsymbol{x}^{\left(j^{\prime}\right)}=\boldsymbol{x}^{(j)}$.

Similarly, not all lights can be jointly turned on. Let $\mathcal{A}=\{1,2, \ldots, l\}$ be the set of lights. For the lights, it is more convenient to work with mutual exclusivity constraints. These can be encoded in an output exclusivity hyper-graph $\mathcal{O}_{\mathcal{A}}:=\left\{\boldsymbol{a}^{(i)} \in\{0,1\}^{l}\right\}_{i=1, \ldots,\left|\mathcal{O}_{\mathcal{A}}\right|}$, with $\left|\mathcal{O}_{\mathcal{A}}\right|=b$, where $i$ labels each exclusivity hyper-edge (one per button). Each $\boldsymbol{a}^{(i)} \in \mathcal{O}_{\mathcal{A}}$ encodes the maximal subset of (mutually exclusive) lights associated with button $i \in \mathcal{X}: a_{k}^{(i)}=0$ stands for " $k$-th light not associated with button $i$ " and $a_{k}^{(i)}=1$ for " $k$-th light associated with button $i$ ". We denote by $\mathcal{A}_{(i)}:=\left\{k \in \mathcal{A}: a_{k}^{(i)}=1\right\}$ the subset of lights (only one of which can be on per run) associated with button $i \in \mathcal{X}$. Accordingly, we denote by $\mathcal{A}_{(\boldsymbol{x})}:=\bigcup_{x_{i}=1, i \in \mathcal{X}} \mathcal{A}_{(i)}$ the subset of lights associated with all the buttons pressed in $\boldsymbol{x} \in \mathcal{I}_{\mathcal{X}}$. In turn, we refer to $\mathcal{X}_{(k)}:=\left\{i \in \mathcal{X}: \boldsymbol{a}_{k}^{(i)}=1\right\}$ as the subset of buttons associated with light $k \in \mathcal{A}$. We restrict throughout to the case in which only incompatible buttons can have common associated lights. That is, we allow that $\left\{i, i^{\prime}\right\} \subseteq \mathcal{X}_{(k)}$ only if $x_{i} \times x_{i^{\prime}}=0$, for all $\boldsymbol{x} \in \mathcal{I}_{\mathcal{X}}$. Finally, we denote by $\boldsymbol{x}_{(k)}:=\left(x_{i}: i \in \mathcal{X}_{(k)}\right)$ the substring of $\boldsymbol{x}$ of buttons associated with light $k$ and by $\boldsymbol{a}_{(\boldsymbol{x})}:=\left(a_{k}: k \in \mathcal{A}_{(\boldsymbol{x})}\right)$ the substring of $\boldsymbol{a}$ of lights associated with the buttons pressed in $\boldsymbol{x}$.

For any input hyper-graph $\mathcal{I}_{\mathcal{X}}$ and output hyper-graph $\mathcal{O}_{\mathcal{A}}$, we consider conditional probability distributions

$$
\boldsymbol{P}_{\mathcal{A} \mid \mathcal{X}}:=\left\{p_{\mathcal{A} \mid \mathcal{X}}(\boldsymbol{a}, \boldsymbol{x})\right\}_{\boldsymbol{a} \in\{0,1\}^{l}, \boldsymbol{x} \in \mathcal{I}_{\mathcal{X}}},
$$

equipped with the property that $p_{\mathcal{A} \mid \mathcal{X}}$ takes positive values for one, and only one, of the lights associated with each pressed button. That is, such that, for each $\boldsymbol{x} \in \mathcal{I}_{\mathcal{X}}, p_{\mathcal{A} \mid \mathcal{X}}(\boldsymbol{a}, \boldsymbol{x}) \neq 0$ only if $\left\|\boldsymbol{a}_{(i)}\right\|_{\mathrm{h}}=1$, with $a_{(i)}=\left(a_{k}: k \in \mathcal{A}_{(i)}\right)$ the substring of $\boldsymbol{a}$ of lights associated with button $i$ and $\left\|\boldsymbol{a}_{(i)}\right\|_{\mathrm{h}}$ the Hamming norm (number of ones in) of $\boldsymbol{a}_{(i)}$, for every $i \in \mathcal{X}$ for which $x_{i}=1$. We refer to any such $\boldsymbol{P}_{\mathcal{A} \mid \mathcal{X}}$ as a box behavior relative to $\mathcal{I}_{\mathcal{X}}$ and $\mathcal{O}_{\mathcal{A}}$. A specially relevant class of behaviors is that of nondisturbing ones: $\boldsymbol{P}_{\mathcal{A} \mid \mathcal{X}}$ is said to be nondisturbing if

$$
\sum_{a_{j}: j \notin \mathcal{A}_{\left(\boldsymbol{x}^{\prime}\right)}} p_{\mathcal{A} \mid \mathcal{X}}(\boldsymbol{a}, \boldsymbol{x})=p_{\mathcal{A}_{\left(\boldsymbol{x}^{\prime}\right)} \mid \mathcal{X}^{\prime}}\left(\boldsymbol{a}_{\left(\boldsymbol{x}^{\prime}\right)}, \boldsymbol{x}^{\prime}\right) \forall \begin{gathered}
\boldsymbol{x} \in \mathcal{I}_{\mathcal{X}}: \boldsymbol{x} \succeq \boldsymbol{x}^{\prime} \\
\boldsymbol{a}_{\left(\boldsymbol{x}^{\prime}\right)}, \boldsymbol{x}^{\prime} \in \mathcal{I}_{\mathcal{X}}
\end{gathered},
$$

where $p_{\mathcal{A}_{\left(\boldsymbol{x}^{\prime}\right)} \mid \mathcal{X}^{\prime}}$ is a conditional probability distribution over $\boldsymbol{a}_{\left(\boldsymbol{x}^{\prime}\right)}$ given $\boldsymbol{x}^{\prime}$. The nondisturbance condition demands that, whenever two contexts have buttons in common, the marginal distribution over the common buttons is independent of the context. It is thus the analogue of the no-signalling condition in Bell scenarios [13].

With this, we can at last provide a precise formal definition of the general mathematical objects of the resource theory. Namely, we call every set of input and output hyper-graphs $\mathcal{I}_{\mathcal{X}}$ and $\mathcal{O}_{\mathcal{A}}$, respectively, together with a nondisturbing behavior $\boldsymbol{P}_{\mathcal{A} \mid \mathcal{X}}$ relative to them, a box

$$
B:=\left\{\mathcal{I}_{\mathcal{X}}, \mathcal{O}_{\mathcal{A}}, \boldsymbol{P}_{\mathcal{A} \mid \mathcal{X}}\right\} .
$$

We call the set of all such nondisturbing boxes ND.

In turn, the free objects of the theory, i.e. the resourceless ones, are given by the class NC $\subset$ ND of noncontextual (NC) boxes, defined by NC box behaviors. A behavior $\boldsymbol{P}_{\mathcal{A} \mid \mathcal{X}}$ is NC if it admits a NC hidden-variable model, i.e., if, for all $\boldsymbol{a} \in\{0,1\}^{|\mathcal{A}|}$ and $\boldsymbol{x} \in \mathcal{I}_{\mathcal{X}}$,

$$
p_{\mathcal{A} \mid \mathcal{X}}(\boldsymbol{a}, \boldsymbol{x})=\sum_{\lambda} p_{\Lambda}(\lambda) \prod_{j \in \mathcal{A}} D_{j}\left(a_{j} \mid \boldsymbol{x}_{(j)}, \lambda\right),
$$

where $\Lambda$ is the hidden variable, taking the value $\lambda$ with probability $p_{\Lambda}(\lambda)$, and $D_{j}\left(a_{j} \mid \boldsymbol{x}_{(j)}, \lambda\right) \quad:=$ $\delta\left(a_{j}, f_{j}\left(\boldsymbol{x}_{(j)}, \lambda\right)\right)$, where $\delta\left(a_{j}, f_{j}\left(\boldsymbol{x}_{(j)}, \lambda\right)\right)$, with $\delta$ the Kronecker delta, is the $\lambda$-th NC deterministic response function for the $j$-th light given the input substring $\boldsymbol{x}_{(j)}$ of buttons associated with light $j$. The function $f_{j}$ encodes the deterministic assignment of $\boldsymbol{x}_{(j)}$ into $a_{j}$ for the $\lambda$-th global deterministic strategy. In particular, it is such that $f_{j}(\mathbf{0}, \lambda)=0$ for all $\lambda$ ( $j$-th light is off if no associated button is pressed, i.e., if $\left.\boldsymbol{x}_{(j)}=\mathbf{0}\right)$. Note that, since $\mathcal{X}_{(j)}$ does not depend on the context, $\boldsymbol{x}_{(j)}$ involves always the same buttons. Furthermore, all these buttons are pressed exclusively in different contexts. This explains why $D_{j}\left(a_{j} \mid \boldsymbol{x}_{(j)}, \lambda\right)$ can only generate NC behaviors in Eq. (4). In fact, one can verify that, when the contexts are defined by space-like separated buttons, expression (4) reduces to the usual local hidden-variable models of Bell nonlocality [13]. Any box outside NC is called contextual. It is a well known fact that measurements on quantum states can yield contextual boxes.

Contextuality-free operations. We consider compositions of the initial box $\boldsymbol{B}$ with a pre-processing box

$$
\boldsymbol{B}_{\mathrm{PRE}}:=\left\{\mathcal{I}_{\mathcal{Y}}, \mathcal{O}_{\mathcal{B}}, \boldsymbol{P}_{\mathcal{B} \mid \mathcal{Y}}\right\} \in \mathrm{NC}
$$


and a $(\boldsymbol{b}, \boldsymbol{y})$-dependent post-processing box

$$
\boldsymbol{B}_{\mathrm{POST}}(\boldsymbol{b}, \boldsymbol{y}):=\left\{\mathcal{I}_{\mathcal{Z}}, \mathcal{O}_{\mathcal{C}}, \boldsymbol{P}_{\mathcal{C} \mid \mathcal{Z}, \boldsymbol{y}, \boldsymbol{b}}\right\} \in \mathrm{NC},
$$

for all $\boldsymbol{b} \in\{0,1\}^{|\mathcal{B}|}$ and $\boldsymbol{y} \in \mathcal{I}_{\mathcal{Y}}$, as shown in Fig. 1. $\mathcal{Y}$ and $\mathcal{B}$ are, respectively, the sets of buttons and lights of $\boldsymbol{B}_{\mathrm{PRE}}$, and $\mathcal{Z}$ and $\mathcal{C}$ those of $\boldsymbol{B}_{\mathrm{POST}}(\boldsymbol{b}, \boldsymbol{y})$. For the composition to be possible, we demand that the set of allowed outputs [inputs] of $\boldsymbol{B}_{\mathrm{PRE}}\left[\boldsymbol{B}_{\mathrm{POST}}(\boldsymbol{b}, \boldsymbol{y})\right]$ is a subset of the set of allowed inputs [outputs] of $\boldsymbol{B}$, i.e., $\overline{\mathcal{O}}_{\mathcal{B}} \subseteq \mathcal{I}_{\mathcal{X}}$ and $\overline{\mathcal{O}}_{\mathcal{A}} \subseteq \mathcal{I}_{\mathcal{Z}}$. Here, we have introduced the complementary hyper-graph $\overline{\mathcal{O}}_{\mathcal{A}}$ to $\mathcal{O}_{\mathcal{A}}$, given by all the output strings with at most one light on per output exclusivity hyper-edge of $\mathcal{O}_{\mathcal{A}}: \overline{\mathcal{O}}_{\mathcal{A}}:=\left\{\boldsymbol{a} \in\{0,1\}|\mathcal{A}|: \forall \boldsymbol{a}^{\prime} \in\right.$ $\left.\mathcal{O}_{\mathcal{A}}, \sum_{k \in \mathcal{A}: a_{k}^{\prime}=1} a_{k} \leq 1\right\}$; and similarly for $\overline{\mathcal{O}}_{\mathcal{B}}$. Thus, $\overline{\mathcal{O}}_{\mathcal{A}}\left(\overline{\mathcal{O}}_{\mathcal{B}}\right)$ gives the combinations of lights on that do not violate any of the exclusivity constraints in $\mathcal{O}_{\mathcal{A}}\left(\mathcal{O}_{\mathcal{B}}\right)$.

Moreover, we allow $\boldsymbol{P}_{\mathcal{C} \mid \mathcal{Z}, \boldsymbol{y}, \boldsymbol{b}}$ to have only a restricted dependence on $(\boldsymbol{b}, \boldsymbol{y})$, in such a way that each output light of the post-processing box is causally influenced only by the inputs and outputs of the pre-processing box that are associated with it. That is, we demand that, for all $\boldsymbol{b} \in\{0,1\}^{|\mathcal{B}|}, \boldsymbol{c} \in\{0,1\}^{|\mathcal{C}|}, \boldsymbol{y} \in \mathcal{I}_{\mathcal{Y}}$, and $\boldsymbol{z} \in \mathcal{I}_{\mathcal{Z}}$,

$$
p_{\mathcal{C} \mid \mathcal{Z}, \boldsymbol{b}, \boldsymbol{y}}(\boldsymbol{c}, \boldsymbol{z})=\sum_{\phi} p_{\Phi}(\phi) \prod_{j \in \mathcal{C}} D_{j}\left(c_{j} \mid \boldsymbol{z}_{(j)}, \boldsymbol{b}_{[j]}, \boldsymbol{y}_{[j]}, \phi\right),
$$

with $D_{j}\left(c_{j} \mid \boldsymbol{z}_{(j)}, \boldsymbol{b}_{[j]}, \boldsymbol{y}_{[j]}, \phi\right)$ defined analogously to $D_{j}\left(a_{j} \mid \boldsymbol{x}_{(j)}, \lambda\right)$ in Eq. (4). As before, $\boldsymbol{z}_{(j)}$ is the substring of $\boldsymbol{z}$ associated with light $j \in \mathcal{C}$, corresponding to the subset $\mathcal{Z}_{(j)} \in \mathcal{Z}$ (of all incompatible buttons). In turn, $\boldsymbol{b}_{[j]}$ and $\boldsymbol{y}_{[j]}$, corresponding $\mathcal{X}_{[j]} \in \mathcal{X}$ and $\mathcal{Y}_{[j]} \in \mathcal{Y}$, respectively, are the substrings of $\boldsymbol{b}$ and $\boldsymbol{y}$ associated with the buttons in $\mathcal{Z}_{(j)}$. More precisely, with $\mathcal{X}_{[j]}:=\left\{\mathcal{X}_{(i)}\right.$ : $\left.i \in \mathcal{Z}_{(j)}\right\}$ and $\mathcal{Y}_{[j]}:=\left\{\mathcal{Y}_{(i)}: i \in \mathcal{X}_{[j]}\right\}$, we explicitly write $\boldsymbol{b}_{[j]}:=\left\{b_{l}: l \in \mathcal{X}_{[j]}\right\}$ and $\boldsymbol{y}_{[j]}:=\left\{y_{l}: l \in \mathcal{Y}_{[j]}\right\}$. In App. A, we show that, for all $j \in \mathcal{C}$, both $\mathcal{X}_{[j]} \in \mathcal{X}$ and $\mathcal{Y}_{[j]} \in \mathcal{Y}$ are composed of mutually incompatible buttons according to $\mathcal{I}_{\mathcal{X}}$ and $\mathcal{I}_{\mathcal{Y}}$, respectively. This is crucial for the composition not to create contextuality.

With this, we are now in a good position to introduce the free operations of contextuality.

Definition 1 (Noncontextual wirings) $W e$ define the noncontextual wiring with respect to pre- and post-processing boxes of Eqs. (5) and (6), respectively, as the linear map $\mathcal{W}_{\mathrm{NC}}$ that takes any initial box $\boldsymbol{B} \in \mathrm{ND}$, given by Eq. (3), into a final box $\boldsymbol{B}_{\mathrm{f}}:=\mathcal{W}_{\mathrm{NC}}(\boldsymbol{B})$ with $b_{\mathrm{f}}:=|\mathcal{Y}|$ buttons and $l_{\mathrm{f}}:=|\mathcal{C}|$ lights, with

$$
\mathcal{W}_{\mathrm{NC}}(\boldsymbol{B}):=\left\{\mathcal{I}_{\mathcal{Y}}, \mathcal{O}_{\mathcal{C}}, \boldsymbol{P}_{\mathcal{C} \mid \mathcal{Y}}\right\},
$$

where $\boldsymbol{P}_{\mathcal{C} \mid \mathcal{Y}}$ is the final behavior, given by

$$
\sum_{\substack{\boldsymbol{\alpha} \in\{0,1\}|\mathcal{A}| \\ \boldsymbol{\beta} \in\{0,1\}^{|\mathcal{B}|}}}^{p_{\mathcal{C} \mid \mathcal{Y}}(\boldsymbol{c}, \boldsymbol{y})=} p_{\mathcal{Z}, \boldsymbol{\beta}, \boldsymbol{y}}(\boldsymbol{c}, \boldsymbol{\alpha}) p_{\mathcal{A} \mid \mathcal{X}}(\boldsymbol{\alpha}, \boldsymbol{\beta}) p_{\mathcal{B} \mid \mathcal{Y}}(\boldsymbol{\beta}, \boldsymbol{y}),
$$

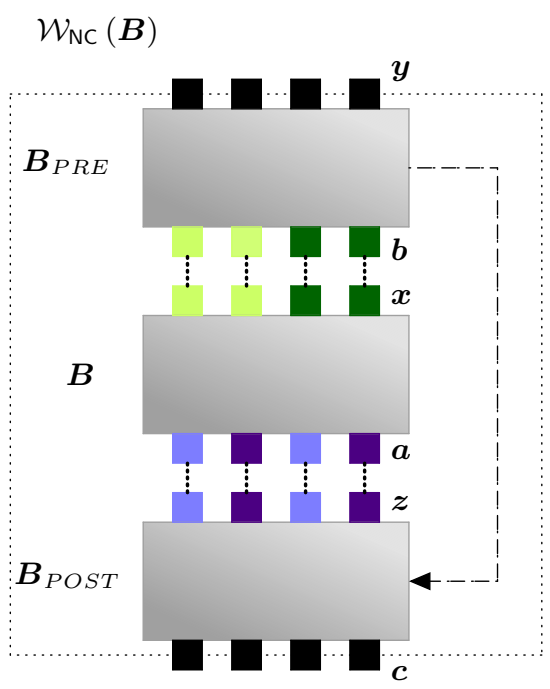

FIG. 1. A noncontextual wiring $\mathcal{W}_{\mathrm{NC}}$ with respect to pre- and post-processing boxes $\boldsymbol{B}_{\mathrm{PRE}}$ and $\boldsymbol{B}_{\mathrm{POST}}$, respectively, maping an initial box $\boldsymbol{B}$ into a final box $\mathcal{W}_{\mathrm{NC}}(\boldsymbol{B})$. The buttons and lights of $\mathcal{W}_{\mathrm{NC}}(\boldsymbol{B})$ are given by the buttons of $\boldsymbol{B}_{\mathrm{PRE}}$ and the lights of $\boldsymbol{B}_{\mathrm{POST}}$, respectively. Only the lights (buttons) of $\boldsymbol{B}$ of the same colour can be on (pressed) at the same time. The behavior of $\boldsymbol{B}_{\mathrm{POST}}$ is causally influenced by $\boldsymbol{B}_{\mathrm{PRE}}$, but in a restricted way such that the statistics of each output light of $\boldsymbol{B}_{\mathrm{POST}}$ depends only on the buttons and lights of $\boldsymbol{B}_{\mathrm{PRE}}$ that are associated with it (see text). As a result, if $\boldsymbol{B}$ is noncontextual so is $\mathcal{W}_{\mathrm{NC}}(\boldsymbol{B})$.

for all $\boldsymbol{c} \in\{0,1\}^{|\mathcal{C}|}$ and $\boldsymbol{y} \in \mathcal{I}_{\mathcal{Y}}$. We denote the class of all such wirings by NCW.

Self-consistency of the theory requires that the class NCW satisfies the following property, proven in App. B.

Lemma 1 (Nondisturbance preservation) The class of boxes ND is closed under all wirings in NCW.

More importantly, to give valid free operations, NCW must fulfill the following requirement, proven in App. C.

Theorem 2 (Noncontextuality preservation) The class of boxes NC is closed under all wirings in NCW.

Intuitively, this is connected to the fact that the composition of any three independent boxes, each one given by a probabilistic mixture of NC deterministic assignments, yields also another such a mixture (with three independent hidden variables). NCW is more general because the pre- and post-processing boxes are not independent. However, the restricted dependence in Eq. (7) enables noncontextuality preservation (see App. C).

Contextuality monotones. In Ref. [14], a measure of contextuality called the relative entropy of contextuality $R_{\mathrm{C}}$ was introduced. For an arbitrary box $\boldsymbol{B} \in \mathrm{ND}$,

$$
R_{\mathrm{C}}(\boldsymbol{B}):=\min _{\boldsymbol{B}^{*} \in \mathrm{NC}} S\left(\boldsymbol{B} \| \boldsymbol{B}^{*}\right) .
$$

$S\left(\boldsymbol{B} \| \boldsymbol{B}^{*}\right)$ is the relative entropy of $\boldsymbol{B}$ with respect to $\boldsymbol{B}^{*}$ (defined precisely in App. D), which measures the distinguishability of $\boldsymbol{B}$ from $\boldsymbol{B}^{*}$ in a broad class of scenarios 


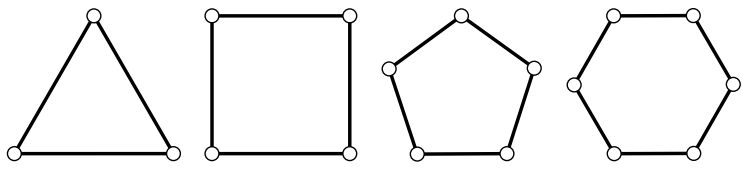

FIG. 2. $b$-cycle graphs $C_{b}$ for $b=3,4,5$, and 6 buttons. A $b$ cycle box is such that the union of all hyper-edges in $\mathcal{I}_{\mathcal{X}}$ equals $C_{b}$ and each input button has its own pair of associated output lights. For even $b$, the class is also intimately connected to the well-known chained inequalities of Bell nonlocality [28]. It includes the Clauser-Horne-Shimony-Holt (CHSH) scenario [29], where the inputs define the square $C_{4}$, and the KlyachkoCan-Binicioğlu-Shumovsky one [30], where the inputs form the pentagon $C_{5}$. For any $b \geq 3$, there exist contextuality bits, i.e., maximally contextual $b$-cycle boxes from which all other $b$-cycle boxes can be obtained for free (see text).

[18]. Hence, $R_{\mathrm{C}}(\boldsymbol{B})$ quantifies the distinguishability of $\boldsymbol{B}$ from its closest (with respect to $S$ ) noncontextual box $\boldsymbol{B}^{*}$, providing a direct generalisation to contextuality of the statistical strength of Bell nonlocality proofs [26].

The essential requirement for a function to be a valid measure of a resource is that it is monotonous (i.e., nonincreasing) under the corresponding free operations. In Ref. [14], the authors show, for all quantum boxes, monotonicity of $R_{\mathrm{C}}$ under probabilistic mixtures of independent channels on each quantum observable (each context). This corresponds to a highly restricted subset of NCW [27]. Here, we show monotonicity of $R_{\mathrm{C}}$ under the whole class NCW and for all boxes $\boldsymbol{B} \in \mathrm{ND}$.

Lemma 3 (Monotonicity of $R_{\mathrm{C}}$ ) Let $\boldsymbol{B} \in \mathrm{ND}$. Then, $R_{\mathrm{C}}\left(\mathcal{W}_{\mathrm{NC}}(\boldsymbol{B})\right) \leq R_{\mathrm{C}}(\boldsymbol{B})$ for all $\mathcal{W}_{\mathrm{NC}} \in \mathrm{NCW}$.

The proof relies explicitly on the parametrization of NCW provided by Eq. (9). It can be found in App. D.

Contextuality bits. The operational framework developed allows us to study contextuality interconversions. A natural question is whether there exists a box from which all boxes, for fixed input and output hyper-graphs, can be obtained for free (i.e., through noncontextual wirings). This is intimately connected to quantification: such a superior box can be taken as unit of contextuality, or contextuality bit, yielding a natural and unambiguous (measure-independent) definition of maximally contextual boxes. Here we answer that question affirmatively for a broad class given by the so-called b-cycle boxes (see Fig. 2). A $b$-cycle box has as many maximal contexts as buttons (b), each $i$-th maximal context consists of two buttons ( $i$ and $i+1$ ), each button belongs to two contexts $\left(x_{i}=1\right.$ for $\boldsymbol{x}^{(i)}$ and $\left.\boldsymbol{x}^{(i-1)}\right)$, and each $i$-th input button has two associated output lights, the $(2 i-1)$-th and the $(2 i)$-th lights, with $l=2 b$. Modulo $b$ is implicitly assumed for the labels of buttons, contexts, and lights. These boxes admit $2^{b-1}$ contextuality bits:

Lemma 4 (Existence of contextuality bits) For any $b \geq 3$, all $b$-cycle boxes in ND can be freely obtained from a b-cycle box with behavior $\boldsymbol{P}_{\mathcal{A} \mid \mathcal{X}}^{(\gamma)}$ of components

$$
p_{\mathcal{A} \mid \mathcal{X}}^{(\gamma)}(\boldsymbol{a}, \boldsymbol{x})= \begin{cases}\frac{1}{2}, & \text { if } x_{i}=1=x_{i+1} \text { and } \\ & a_{2 i-\gamma_{i}}=a_{2(i+1)-\gamma_{i+1}} \\ 0, & \text { otherwise }\end{cases}
$$

for all $i \in \mathcal{X}$, with $\gamma:=\left(\gamma_{1}, \ldots, \gamma_{b}\right)$ such that $\gamma_{i}=0$ or 1 and $\|\gamma\|_{\mathrm{h}}$ is an odd integer.

Eq. (11) describes any of the $2^{b-1}$ contextual $b$-cycle behaviors extremal in ND, derived (in a different notation) and shown to be equivalent under noncontextual relabelings of outputs in Ref. [31]. The proof of the lemma, given in App. E, consists then in showing that any convex combination of noncontextual relabelings of outputs can be carried out by a wiring in NCW. For the particular case $b=4$ (the CHSH scenario), the behaviors in Eq. (11) become equivalent to the no-signalling extremal Popescu-Rohrlich box [32], which is known to generate all other no-signalling boxes under local wirings assisted by shared randomness [16-18]. Lemma 4 thus generalizes this fact to arbitrary $b \geq 3$ and noncontextual wirings. Finally, it is important to mention that, for even $b$, the buttons can be split into two disjoint subsets of $b / 2$ incompatible buttons each, an the lights can be reduced from $2 b$ to only 4 (one mutually exclusive pair per subset of buttons), as in the chained inequalities [28]. This is an alternative representation of the same physical box. Our formalism is totally versatile in this sense, as it can directly deal with any chosen representation of a box.

Final discussion. In contrast to more abstract approaches $[14,15]$, noncontextual wirings admit a friendly analytical parametrization. This is useful to classify, quantify, and operationally manipulate contextuality as a formal resource. For instance, monotonicity of contextuality under a non-trivial class of free operations was not clear for a long time. Here, we have solved this problem by showing that the relative entropy of contextuality is a contextuality monotone under noncontextual wirings. Furthermore, we have also shown that single, maximallycontextual boxes that serve as contextuality bits exist for all cycle boxes. Cycle boxes encompass important Bell scenarios [29, 30]; and the treatment can additionally be extended to bipartite boxes with more outputs [21]. Interesting open questions are, e.g., what the simplest box admitting inequivalent (i.e, not freely interconvertible) classes of contextuality is and what the simplest one allowing for contextuality distillation. Our findings provide the missing ingredient for a complete, unified resource theory of contextuality and Bell nonlocality.

Acknowledgements. The present work was initiated during the workshop "Quantum Correlations, Contextuality, and All That... Again" at the International Institute of Physics (IIP), Natal, Brazil. The participants of the workshop as well as the hospitality of IIP are gratefully acknowledged. We thank D. Cavalcanti, C. Duarte, and M. Pusey for fruitful discussions. BA thanks the Instituto de Matemática Pura e Aplicada (IMPA) for the 
hospitality at Rio de Janeiro, Brazil. BA, MTC, and LA acknowledge financial support from the Brazilian ministries MEC and MCTIC and agencies CNPq, CAPES, FAPERJ, FAEPEX, and INCT-IQ. AC acknowledges support from Project No. FIS2014-60843-P, "Advanced
Quantum Information" (MINECO, Spain), with FEDER funds, the FQXi Large Grant "The Observer Observed: A Bayesian Route to the Reconstruction of Quantum Theory," and the project "Photonic Quantum Information" (Knut and Alice Wallenberg Foundation, Sweden).
[1] E. P. Specker, Die Logik nicht gleichzeitig entscheidbarer Aussagen, Dialectica 14, 239 (1960) [The logic of nonsimultaneously decidable propositions, arXiv:1103.4537].

[2] S. Kochen and E. P. Specker, The problem of hidden variables in quantum mechanics, J. Math. Mech. 17, 59 (1967).

[3] J. S. Bell, On the problem of hidden variables in quantum mechanics, Rev. Mod. Phys. 38, 447 (1966).

[4] Y. Hasegawa, R. Loidl, G. Badurek, M. Baron, and H. Rauch, Quantum Contextuality in a Single-Neutron Optical Experiment, Phys. Rev. Lett. 97, 230401 (2006).

[5] G. Kirchmair, F. Zähringer, R. Gerritsma, M. Kleinmann, O. Gühne, A. Cabello, R. Blatt, and C. F. Roos, State-independent experimental test of quantum contextuality, Nature (London) 460, 494 (2009).

[6] E. Amselem, M. Rådmark, M. Bourennane, and A. Cabello, State-Independent Quantum Contextuality with Single Photons, Phys. Rev. Lett. 103, 160405 (2009).

[7] R. Eapkiewicz, P. Li, C. Schaeff, N. Langford, S. Ramelow, M. Wieśniak, and A. Zeilinger, Experimental non-classicality of an indivisible quantum system, Nature (London) 474, 490 (2011).

[8] G. Borges, M. Carvalho, P.-L. de Assis, J. Ferraz, M. Araújo, A. Cabello, M. Terra Cunha, and S. Pádua, Quantum contextuality in a Young-type interference experiment, Phys. Rev. A 89, 052106 (2014).

[9] M. Howard, J. J. Wallman, V. Veitch, and J. Emerson, Contextuality supplies the 'magic' for quantum computation, Nature (London) 510, 351 (2014).

[10] R. Raussendorf, Contextuality in measurement-based quantum computation, Phys. Rev. A 88, 022322 (2013).

[11] N. Delfosse, P. A. Guerin, J. Bian, and R. Raussendorf, Wigner function negativity and contextuality in quantum computation on rebits, Phys. Rev. X 5, 021003 (2015).

[12] M. Um, Mark, X. Zhang, J. Zhang, Y. Wang, S. Yangchao, D. Deng, L. Duan, and K. Kim, Experimental certification of random numbers via quantum contextuality, Sci. Rep. 3, 1627 (2013).

[13] N. Brunner, D. Cavalcanti, S. Pironio, V. Scarani, and S. Wehner, Bell nonlocality, Rev. Mod. Phys. 86, 419 (2014).

[14] A. Grudka, K. Horodecki, M. Horodecki, P. Horodecki, R. Horodecki, P. Joshi, W. Kłobus, and A. Wójcik, Quantifying Contextuality, Phys. Rev. Lett. 112, 120401 (2014).

[15] K. Horodecki, A. Grudka, P. Joshi, W. Kłobus, and J. Eodyga, Axiomatic approach to contextuality and nonlocality, Phys. Rev. A 92, 032104 (2015).

[16] R. Gallego, L. E. Würflinger, A. Acín, and M. Navascués, Operational Framework for Nonlocality, Phys. Rev. Lett. 109, 070401 (2012).

[17] J. I. de Vicente, On nonlocality as a resource theory and nonlocality measures, J. Phys. A: Math. Theor. 47, 424017 (2014).
[18] R. Gallego and L. Aolita, Nonlocality free wirings and the distinguishability between Bell boxes, Phys. Rev. A 95, 032118 (2017).

[19] F. G. S. L. Brandão and G. Gour, Reversible Framework for Quantum Resource Theories, Phys. Rev. Lett. 115, 070503 (2015); Phys. Rev. Lett. 115, 199901 (2015).

[20] B. Coecke, T. Fritz, and R. W. Spekkens, A mathematical theory of resources, Inf. Comput. 250, 59 (2016).

[21] J. Barrett, N. Linden, S. Massar, S. Pironio, S. Popescu, and D. Roberts, Nonlocal correlations as an informationtheoretic resource, Phys. Rev. A 71, 022101 (2005).

[22] J. Allcock, N. Brunner, N. Linden, S. Popescu, P. Skrzypczyk, and T. Vértesi, Closed sets of nonlocal correlations, Phys. Rev. A 80, 062107 (2009).

[23] P. Joshi, M. Horodecki, R. Horodecki, A. Grudka, K. Horodecki, and P. Horodecki, No-broadcasting of nonsignaling boxes via operations which transform local boxes into local ones, Quantum Info. Comput. 13, 567 (2013).

[24] B. Lang, T. Vértesi, and M. Navascués, Closed sets of correlations: answers from the zoo, J. Phys. A: Math. Theor. 47, 424029 (2014).

[25] R. Gallego and L. Aolita, Resource theory of steering, Phys. Rev. X 5, 041008 (2015).

[26] W. van Dam, R. D. Gill, and P. D. Grünwald, The statistical strength of nonlocality proofs, IEEE Trans. Inf. Theory 51, 2812 (2005).

[27] In fact, in Ref. [14], monotonicity of another related quantity is shown, the uniform relative entropy of contextuality, under the same restricted subclass. However, one can show that the uniform variant is not monotonous under more general wirings in NCW, even restricted to trivial (identity) post-processing boxes [18].

[28] S. L. Braunstein and C. M. Caves, Wringing out better Bell inequalities, Ann. Phys. 202, 22 (1990).

[29] J. F. Clauser, M. A. Horne, A. Shimony, and R. A. Holt, Proposed Experiment to Test Local Hidden-Variable Theories, Phys. Rev. Lett. 23, 880 (1969).

[30] A. A. Klyachko, M. A. Can, S. Binicioğlu, and A. S. Shumovsky, Simple Test for Hidden Variables in Spin-1 Systems, Phys. Rev. Lett. 101, 020403 (2008).

[31] M. Araújo, M. T. Quintino, C. Budroni, M. Terra Cunha, and A. Cabello, All noncontextuality inequalities for the n-cycle scenario, Phys. Rev. 88, 022118 (2013).

[32] S. Popescu and D. Rohrlich, Quantum nonlocality as an axiom, Found. Phys. 24, 379 (1994).

Appendix A: Proof that the buttons in $\mathcal{X}_{[j]} \in \mathcal{X}$ and $\mathcal{Y}_{[j]}$ are all incompatible

Here, we explicitly prove that for all $j \in \mathcal{C}$, both $\mathcal{X}_{[j]} \in \mathcal{X}$ and $\mathcal{Y}_{[j]} \in \mathcal{Y}$, as defined right after Eq. (7), are 
composed exclusively of incompatible buttons according to $\mathcal{I}_{\mathcal{X}}$ and $\mathcal{I}_{\mathcal{Y}}$. The proof is simple and consists of reductio ad absurdum. Suppose that, for some $j \in \mathcal{C}$, not all buttons in $\mathcal{X}_{[j]}$ are incompatible. This implies that the subset $\mathcal{A}_{[j]}$ of lights in $\mathcal{A}$ associated with $j \in \mathcal{C}$ are not all mutually exclusive. Since $\mathcal{A}_{[j]}$ coincides with the subset of buttons $\mathcal{Z}_{(j)}$, that implies, in turn, that not all buttons in $\mathcal{Z}_{(j)}$ are incompatible. However, the latter is false by assumption (because $\boldsymbol{B}_{\mathrm{POST}}(\boldsymbol{b}, \boldsymbol{y})$ are welldefined boxes, so that no compatible buttons can share a common associated light). This proves that $\mathcal{X}_{[j]} \in \mathcal{X}$ contains always only incompatible buttons according to $\mathcal{I}_{\mathcal{X}}$. By the same reasoning, this implies that $\mathcal{Y}_{[j]} \in \mathcal{Y}$ contains always only incompatible according to $\mathcal{I}_{\mathcal{Y}}$, which finishes the proof.

\section{Appendix B: Proof of Lemma 1}

For any $\boldsymbol{y}, \boldsymbol{w} \in \mathcal{I}_{\mathcal{Y}}$ such that $\boldsymbol{y} \succeq \boldsymbol{w}$, consider the sum

$$
\sum_{c_{j} ; j \notin \mathcal{C}[\boldsymbol{w}]} p_{\mathcal{C} \mid \mathcal{Z}, \boldsymbol{b}, \boldsymbol{y}}(\boldsymbol{c}, \boldsymbol{z})
$$

in which $\mathcal{C}_{[\boldsymbol{w}]}$ is the set of lights in $\mathcal{C}$ associated with the buttons in $\boldsymbol{w}$ for the resulting wired box $\mathcal{W}_{\mathrm{NC}}(\boldsymbol{B})$. Let $\tilde{\boldsymbol{a}}=\boldsymbol{a}_{(\boldsymbol{w})}$ and $\tilde{\boldsymbol{b}}=\boldsymbol{b}_{(\tilde{\boldsymbol{a}})}$. For $j \in \mathcal{C}_{[\boldsymbol{w}]}$, we have that $\boldsymbol{a}_{(j)}=\tilde{\boldsymbol{a}_{(j)}}, \boldsymbol{b}_{[j]}=\tilde{\boldsymbol{b}_{[j]}}$, and $\boldsymbol{y}_{[j]}=\tilde{\boldsymbol{y}_{[j]}}$, otherwise we would have incompatible buttons in the same context, which is not possible. Hence, Eq. (7) implies that

$$
\sum_{c_{j} ; j \notin \mathcal{C}_{[\boldsymbol{w}]}} p_{\mathcal{C} \mid \mathcal{Z}, \boldsymbol{b}, \boldsymbol{y}}(\boldsymbol{c}, \boldsymbol{a})=p_{\mathcal{C} \mid \mathcal{Z}, \tilde{\boldsymbol{b}}, \boldsymbol{w}}\left(\boldsymbol{c}_{[w]}, \tilde{\boldsymbol{a}}\right) .
$$

From this it follows that

$$
\begin{aligned}
\sum_{c_{j} ; j \notin \mathcal{C}_{[w]}} p_{\mathcal{C} \mid \mathcal{Y}}(\boldsymbol{c}, \boldsymbol{y}) & =\sum_{c_{j} ; j \notin \mathcal{C}_{[\boldsymbol{w}]}} \sum_{\boldsymbol{\alpha}, \boldsymbol{\beta}} p_{\mathcal{C} \mid \mathcal{Z}, \boldsymbol{b}, \boldsymbol{y}}(\boldsymbol{c}, \boldsymbol{\alpha}) p_{\mathcal{A} \mid \mathcal{X}}(\boldsymbol{\alpha}, \boldsymbol{\beta}) p_{\mathcal{B} \mid \mathcal{Y}}(\boldsymbol{\beta}, \boldsymbol{y}) \\
& =\sum_{\boldsymbol{\alpha}, \boldsymbol{\beta}} p_{\mathcal{C} \mid \mathcal{Z}, \tilde{\boldsymbol{b}}, \boldsymbol{w}}\left(\boldsymbol{c}_{[w]}, \tilde{\boldsymbol{\alpha}}\right) p_{\mathcal{A} \mid \mathcal{X}}(\boldsymbol{\alpha}, \boldsymbol{\beta}) p_{\mathcal{B} \mid \mathcal{Y}}(\boldsymbol{\beta}, \boldsymbol{y}) \\
& =\sum_{\tilde{\boldsymbol{\alpha}}, \tilde{\boldsymbol{\beta}}} p_{\mathcal{C} \mid \mathcal{Z}, \tilde{\boldsymbol{\beta}}, \boldsymbol{y}}\left(\boldsymbol{c}_{[w]}, \tilde{\boldsymbol{\alpha}}\right) p_{\mathcal{A} \mid \mathcal{X}}(\tilde{\boldsymbol{\alpha}}, \tilde{\boldsymbol{\beta}}) p_{\mathcal{B} \mid \mathcal{Y}}(\tilde{\boldsymbol{\beta}}, \tilde{\boldsymbol{y}}) \\
& =p_{\mathcal{C}_{[w]} \mid \mathcal{Y}}\left(\boldsymbol{c}_{[w]}, \boldsymbol{w}\right) .
\end{aligned}
$$

This concludes the proof that the resulting wired box $\mathcal{W}_{\mathrm{NC}}(\boldsymbol{B})$ is nondisturbing.

\section{Appendix C: Proof of Theorem 2}

Assuming Eqs. (4) and (7), and the analogous equation for $\boldsymbol{B}_{\mathrm{PRE}}$ : for all $\boldsymbol{b} \in\{0,1\}^{\mathcal{B}}$ and $\boldsymbol{y} \in \mathcal{I}_{\mathcal{Y}}$,

$$
p_{\mathcal{B} \mid \mathcal{Y}}(\boldsymbol{b}, \boldsymbol{y})=\sum_{\gamma} p_{\Gamma}(\gamma) \prod_{j \in \mathcal{B}} D_{j}\left(b_{j} \mid \boldsymbol{y}_{(j)}, \gamma\right),
$$

we need to prove that the final behavior in Eq. (9) is noncontextual.

$$
\begin{aligned}
p_{\mathcal{C} \mid \mathcal{Y}}(\boldsymbol{c}, \boldsymbol{y})= & \sum_{\substack{\boldsymbol{\alpha} \in\{0,1\}^{|\mathcal{A}|} \\
\boldsymbol{\beta} \in\{0,1\}^{|\mathcal{B}|}}} p_{\mathcal{C} \mid \mathcal{Z}, \boldsymbol{\beta}, \boldsymbol{y}}(\boldsymbol{c}, \boldsymbol{\alpha}) p_{\mathcal{A} \mid \mathcal{X}}(\boldsymbol{\alpha}, \boldsymbol{\beta}) p_{\mathcal{B} \mid \mathcal{Y}}(\boldsymbol{\beta}, \boldsymbol{y}) \\
= & \sum_{\substack{\boldsymbol{\alpha} \in\{0,1\}^{|\mathcal{A}|} \\
\boldsymbol{\beta} \in\{0,1\}^{|\mathcal{B}|}}} \sum_{\boldsymbol{\lambda}} p_{\boldsymbol{\Lambda}}(\boldsymbol{\lambda}) \prod_{j \in \mathcal{C}} D_{j}\left(c_{j} \mid \boldsymbol{\alpha}_{(j)}, \boldsymbol{\beta}_{[j]}, \boldsymbol{y}_{[j]}, \phi\right) \prod_{k \in \mathcal{A}} D_{k}\left(\alpha_{k} \mid \boldsymbol{\beta}_{(k)}, \lambda\right) \prod_{i \in \mathcal{B}} D_{i}\left(\beta_{i} \mid \boldsymbol{y}_{(i)}, \gamma\right) \\
= & \sum_{\boldsymbol{\lambda}} p_{\boldsymbol{\Lambda}}(\boldsymbol{\lambda}) \prod_{j \in \mathcal{C}} D_{j}\left(c_{j} \mid \tilde{\boldsymbol{\alpha}}_{(j)}\left(\tilde{\boldsymbol{\beta}}_{[j]}\left(\boldsymbol{y}_{[j]}, \gamma\right), \lambda\right), \tilde{\boldsymbol{\beta}}_{[j]}\left(\boldsymbol{y}_{[j]}, \gamma\right), \boldsymbol{y}_{[j]}, \phi\right)
\end{aligned}
$$

where the sums over $\boldsymbol{a}$ and $\boldsymbol{b}$ disappear because of the deterministic response functions. Besides, $\tilde{\boldsymbol{\beta}}_{[j]}\left(\boldsymbol{y}_{[j]}, \gamma\right)$ is

To this end, let us first introduce the short-hand notation $\boldsymbol{\lambda}:=(\gamma, \lambda, \phi)$ and $p_{\boldsymbol{\Lambda}}(\boldsymbol{\lambda}):=p_{\Gamma}(\gamma) p_{\Lambda}(\lambda) p_{\Phi}(\phi)$ Then, we explicitly write out Eq. (9) as 
the deterministic output substring of the pre-processing box as a function of the input substring $\boldsymbol{y}$ and the deterministic strategy $\gamma$, and $\tilde{\boldsymbol{\alpha}}_{(j)}\left(\tilde{\boldsymbol{\beta}}_{[j]}\left(\boldsymbol{y}_{[j]}, \gamma\right), \lambda\right)$ is the deterministic output substring of the initial box as a function of the input substring $\tilde{\boldsymbol{\beta}}_{[j]}\left(\boldsymbol{y}_{[j]}, \gamma\right)$ and the deterministic strategy $\lambda$.

Identifying the $\lambda$-th $\mathrm{NC}$ deterministic response function for the output light $j \in \mathcal{C}$ given the input substring of buttons in $\mathcal{Y}$ associated with light $j \in \mathcal{C}$ as $D_{j}\left(c_{j} \mid \boldsymbol{y}_{[j]}, \boldsymbol{\lambda}\right):=$ $D_{j}\left(c_{j} \mid \tilde{\boldsymbol{\alpha}}_{(j)}\left(\tilde{\boldsymbol{\beta}}_{[j]}\left(\boldsymbol{y}_{[j]}, \gamma\right), \lambda\right), \tilde{\boldsymbol{\beta}}_{[j]}\left(\boldsymbol{y}_{[j]}, \gamma\right), \boldsymbol{y}_{[j]}, \phi\right), \quad$ we write Eq. (C2) as

$$
\begin{aligned}
p_{\mathcal{C} \mid \mathcal{Y}}(\boldsymbol{c}, \boldsymbol{y}) & =\sum_{\boldsymbol{\lambda}} p_{\boldsymbol{\Lambda}}(\boldsymbol{\lambda}) D(\boldsymbol{c} \mid \boldsymbol{\lambda}, \boldsymbol{y}) \\
& =\sum_{\boldsymbol{\lambda}} p_{\boldsymbol{\Lambda}}(\boldsymbol{\lambda}) \prod_{j \in \mathcal{C}} D_{j}\left(c_{j} \mid \boldsymbol{y}_{[j]}, \boldsymbol{\lambda}\right)
\end{aligned}
$$

which is manifestly in the explicit form of a NC hiddenvariable model. This concludes the proof.

\section{Appendix D: Proof of Lemma 3}

We begin with the definition of $S\left(\boldsymbol{B} \| \boldsymbol{B}^{*}\right)$ appearing in Eq. (10). The relative entropy, or Kullback-Leibler divergence, $S_{\mathrm{d}}$ of a probability distribution $\boldsymbol{P}$ (over a sample space $\Omega$ ) relative to another probability distribution $\boldsymbol{P}^{*}$ (over the same sample space) is defined as

$$
S_{\mathrm{d}}\left(\boldsymbol{P} \| \boldsymbol{P}^{*}\right)=\sum_{i \in \Omega} p(i) \log \left(\frac{p(i)}{p^{*}(i)}\right) .
$$

With this, one can define the relative entropy $S_{\mathrm{b}}$ between two behaviors $\boldsymbol{P}_{\mathcal{A} \mid \mathcal{X}}$ and $\boldsymbol{P}_{\mathcal{A} \mid \mathcal{X}}^{*}$ as the relative entropy $S_{\mathrm{d}}$ between the output distributions obtained from $\boldsymbol{P}_{\mathcal{A} \mid \mathcal{X}}$ and $\boldsymbol{P}_{\mathcal{A} \mid \mathcal{X}}^{*}$ for the optimal input choice [18]:

$$
S_{\mathrm{b}}\left(\boldsymbol{P}_{\mathcal{A} \mid \mathcal{X}} \| \boldsymbol{P}_{\mathcal{A} \mid \mathcal{X}}^{*}\right):=\max _{\boldsymbol{x} \in \mathcal{I}_{\mathcal{X}}} S_{\mathrm{d}}\left(\boldsymbol{P}_{\mathcal{A} \mid \mathcal{X}}(\cdot, \boldsymbol{x}) \| \boldsymbol{P}_{*} \mathcal{A} \mid \mathcal{X}(\cdot, \boldsymbol{x})\right) .
$$

In turn, the (box) relative entropy $S$ between two nondisturbing boxes $\boldsymbol{B}:=\left\{\mathcal{I}_{\mathcal{X}}, \mathcal{O}_{\mathcal{A}}, \boldsymbol{P}_{\mathcal{A} \mid \mathcal{X}}\right\}$ and $\boldsymbol{B}^{*}:=$ $\left\{\mathcal{I}_{\mathcal{X}}, \mathcal{O}_{\mathcal{A}}, \boldsymbol{P}_{\mathcal{A} \mid \mathcal{X}}^{*}\right\}$ is defined as the (behavior) relative entropy between their respective behaviors [14]:

$$
S\left(\boldsymbol{B} \| \boldsymbol{B}^{*}\right):=S_{\mathrm{b}}\left(\boldsymbol{P}_{\mathcal{A} \mid \mathcal{X}} \| \boldsymbol{P}_{\mathcal{A} \mid \mathcal{X}}^{*}\right) .
$$

Now we proceed to prove the lemma. First we show monotonicity of the box relative entropy $S$ under an arbitrary noncontextual wiring $\mathcal{W}_{\mathrm{NC}} \in \mathrm{NC}$. Given $\boldsymbol{B}:=$ $\left\{\mathcal{I}_{\mathcal{X}}, \mathcal{O}_{\mathcal{A}}, \boldsymbol{P}_{\mathcal{A} \mid \mathcal{X}}\right\} \in \mathrm{NC}$ and $\boldsymbol{B}^{\prime}:=\left\{\mathcal{I}_{\mathcal{X}}, \mathcal{O}_{\mathcal{A}}, \boldsymbol{P}_{\mathcal{A} \mid \mathcal{X}}^{\prime}\right\} \in$ NC, let $\boldsymbol{B}_{\mathrm{f}}:=\mathcal{W}_{\mathrm{NC}}(\boldsymbol{B})=\left\{\mathcal{I}_{\mathcal{Y}}, \mathcal{O}_{\mathcal{C}}, \boldsymbol{P}_{\mathcal{C} \mid \mathcal{Y}}\right\}$ and $\boldsymbol{B}_{\mathrm{f}}^{\prime}:=$ $\mathcal{W}_{\mathrm{NC}}\left(\boldsymbol{B}^{\prime}\right)=\left\{\mathcal{I}_{\mathcal{Y}}, \mathcal{O}_{\mathcal{C}}, \boldsymbol{P}_{\mathcal{C} \mid \mathcal{Y}}^{\prime}\right\}$. In addition, denote by $\boldsymbol{y}^{*}$ the string in $\mathcal{I}_{\mathcal{Y}}$ such that

$$
\begin{aligned}
& S_{\mathrm{d}}\left(\boldsymbol{P}_{\mathcal{C} \mid \mathcal{Y}}\left(\cdot, \boldsymbol{y}^{*}\right) \| \boldsymbol{P}_{\mathcal{C} \mid \mathcal{Y}}^{\prime}\left(\cdot, \boldsymbol{y}^{*}\right)\right):= \\
& \max _{\boldsymbol{y} \in \mathcal{I}_{\mathcal{Y}}} S_{\mathrm{d}}\left(\boldsymbol{P}_{\mathcal{C} \mid \mathcal{Y}}(\cdot, \boldsymbol{y}) \| \boldsymbol{P}_{\mathcal{C} \mid \mathcal{Y}}^{\prime}(\cdot, \boldsymbol{y})\right) .
\end{aligned}
$$

Then,

$$
\begin{aligned}
S\left(\boldsymbol{B}_{\mathrm{f}} \| \boldsymbol{B}_{\mathrm{f}}^{\prime}\right)= & \max _{\boldsymbol{y} \in \mathcal{I}_{\mathcal{Y}}} S_{\mathrm{d}}\left(\boldsymbol{P}_{\mathcal{C} \mid \mathcal{Y}}(\cdot, \boldsymbol{y}) \| \boldsymbol{P}_{\mathcal{C} \mid \mathcal{Y}}^{\prime}(\cdot, \boldsymbol{y})\right\} \\
= & \sum_{\boldsymbol{c}} p_{\mathcal{C} \mid \mathcal{Y}}\left(\boldsymbol{c}, \boldsymbol{y}^{*}\right) \log \left(\frac{p_{\mathcal{C} \mid \mathcal{Y}}\left(\boldsymbol{c}, \boldsymbol{y}^{*}\right)}{p_{\mathcal{C} \mid \mathcal{Y}}^{\prime}\left(\boldsymbol{c}, \boldsymbol{y}^{*}\right)}\right) \\
= & \sum_{\boldsymbol{\alpha}, \boldsymbol{\beta}, \boldsymbol{c}} p_{\mathcal{C} \mid \mathcal{Z}, \boldsymbol{\beta}, \boldsymbol{y}^{*}}(\boldsymbol{c}, \boldsymbol{\alpha}) p_{\mathcal{A} \mid \mathcal{X}}(\boldsymbol{\alpha}, \boldsymbol{\beta}) p_{\mathcal{B} \mid \mathcal{Y}}\left(\boldsymbol{\beta}, \boldsymbol{y}^{*}\right) \log \left(\frac{\left.\sum_{\boldsymbol{\alpha}, \boldsymbol{\beta}} p_{\mathcal{C} \mid \mathcal{Z}, \boldsymbol{\beta}, \boldsymbol{y}^{*}}(\boldsymbol{c}, \boldsymbol{\alpha}) p_{\mathcal{A} \mid \mathcal{X}}(\boldsymbol{\alpha}, \boldsymbol{\beta}) p_{\mathcal{B} \mid \mathcal{Y}}\left(\boldsymbol{\beta}, \boldsymbol{y}^{*}\right)\right)}{\sum_{\boldsymbol{\alpha}, \boldsymbol{\beta}} p_{\mathcal{C} \mid \mathcal{Z}, \boldsymbol{\beta}, \boldsymbol{y}^{*}}(\boldsymbol{c}, \boldsymbol{\alpha}) p_{\mathcal{A} \mid \mathcal{X}}^{\prime}(\boldsymbol{\alpha}, \boldsymbol{\beta}) p_{\mathcal{B} \mid \mathcal{Y}}\left(\boldsymbol{\beta}, \boldsymbol{y}^{*}\right)}\right) \\
\leq & \sum_{\boldsymbol{\alpha}, \boldsymbol{\beta}, \boldsymbol{c}} p_{\mathcal{C} \mid \mathcal{Z}, \boldsymbol{\beta}, \boldsymbol{y}^{*}}(\boldsymbol{c}, \boldsymbol{\alpha}) p_{\mathcal{A} \mid \mathcal{X}}(\boldsymbol{\alpha}, \boldsymbol{\beta}) p_{\mathcal{B} \mid \mathcal{Y}}\left(\boldsymbol{\beta}, \boldsymbol{y}^{*}\right) \log \left(\frac{p_{\mathcal{C} \mid \mathcal{Z}, \boldsymbol{\beta}, \boldsymbol{y}^{*}}(\boldsymbol{c}, \boldsymbol{\alpha}) p_{\mathcal{A} \mid \mathcal{X}}(\boldsymbol{\alpha}, \boldsymbol{\beta}) p_{\mathcal{B} \mid \mathcal{Y}}\left(\boldsymbol{\beta}, \boldsymbol{y}^{*}\right)}{p_{\mathcal{C} \mid \mathcal{Z}, \boldsymbol{\beta}, \boldsymbol{y}^{*}}(\boldsymbol{c}, \boldsymbol{\alpha}) p_{\mathcal{A} \mid \mathcal{X}}^{\prime}(\boldsymbol{\alpha}, \boldsymbol{\beta}) p_{\mathcal{B} \mid \mathcal{Y}}\left(\boldsymbol{\beta}, \boldsymbol{y}^{*}\right)}\right) \\
= & \sum_{\boldsymbol{\alpha}, \boldsymbol{\beta}, \boldsymbol{c}} p_{\mathcal{C} \mid \mathcal{Z}, \boldsymbol{\beta}, \boldsymbol{y}^{*}}(\boldsymbol{c}, \boldsymbol{\alpha}) p_{\mathcal{A} \mid \mathcal{X}}(\boldsymbol{\alpha}, \boldsymbol{\beta}) p_{\mathcal{B} \mid \mathcal{Y}}\left(\boldsymbol{\beta}, \boldsymbol{y}^{*}\right) \log \left(\frac{p_{\mathcal{A} \mid \mathcal{X}}(\boldsymbol{\alpha}, \boldsymbol{\beta})}{p_{\mathcal{A} \mid \mathcal{X}}^{\prime}(\boldsymbol{\alpha}, \boldsymbol{\beta})}\right) \\
= & \sum_{\boldsymbol{\alpha}, \boldsymbol{\beta}} p_{\mathcal{A} \mid \mathcal{X}}(\boldsymbol{\alpha}, \boldsymbol{\beta}) p_{\mathcal{B} \mid \mathcal{Y}}\left(\boldsymbol{\beta}, \boldsymbol{y}^{*}\right) \log \left(\frac{p_{\mathcal{A} \mid \mathcal{X}}(\boldsymbol{\alpha}, \boldsymbol{\beta})}{p_{\mathcal{A} \mid \mathcal{X}}^{\prime}(\boldsymbol{\alpha}, \boldsymbol{\beta})}\right)
\end{aligned}
$$




$$
\begin{aligned}
& =\sum_{\boldsymbol{\beta}} p_{\mathcal{B} \mid \mathcal{Y}}\left(\boldsymbol{\beta}, \boldsymbol{y}^{*}\right) \sum_{\boldsymbol{\alpha}} p_{\mathcal{A} \mid \mathcal{X}}(\boldsymbol{\alpha}, \boldsymbol{\beta}) \log \left(\frac{p_{\mathcal{A} \mid \mathcal{X}}(\boldsymbol{\alpha}, \boldsymbol{\beta})}{p_{\mathcal{A} \mid \mathcal{X}}^{\prime}(\boldsymbol{\alpha}, \boldsymbol{\beta})}\right) \\
& =\sum_{\boldsymbol{\beta}} p_{\mathcal{B} \mid \mathcal{Y}}\left(\boldsymbol{\beta}, \boldsymbol{y}^{*}\right) S_{\mathrm{d}}\left(\boldsymbol{P}_{\mathcal{A} \mid \mathcal{X}}(\cdot, \boldsymbol{\beta}) \| \boldsymbol{P}_{\mathcal{A} \mid \mathcal{X}}^{\prime}(\cdot, \boldsymbol{\beta})\right) \\
& \leq \max _{\boldsymbol{x} \in \mathcal{I}_{\mathcal{X}}} S_{\mathrm{d}}\left(\boldsymbol{P}_{\mathcal{A} \mid \mathcal{X}}(\cdot, \boldsymbol{x}) \| \boldsymbol{P}_{\mathcal{A} \mid \mathcal{X}}^{\prime}(\cdot, \boldsymbol{x})\right) \\
& =S\left(\boldsymbol{B} \| \boldsymbol{B}^{\prime}\right) .
\end{aligned}
$$

Eqs. (D5) follows from the definition of $S$, Eq. (D6) from Eq. (D4), Eq. (D7) from Eq. (9), Eq. (D8) from the log sum inequality $\sum_{i} x_{i} \log \left(\frac{\sum_{i} x_{i}}{\sum_{i} y_{i}}\right) \leq \sum_{i} x_{i} \log \left(\frac{x_{i}}{y_{i}}\right)$, Eqs. (D9) and (D11) from basic algebra, Eq. (D10) from summing over $\boldsymbol{c}$ and the fact that $p_{\mathcal{C} \mid \mathcal{Z}, \boldsymbol{\beta}, \boldsymbol{y}}(\cdot, \boldsymbol{\alpha})$ is a wellnormalized probability distribution, Eqs. (D12) from the definition of $S_{\mathrm{b}}$, Eq. (D13) from the fact the average is smaller than the largest value, and Eq. (D14) from the definition of $S_{\mathrm{d}}$.

Now we can prove monotonicity of $R_{C}$. Let $\boldsymbol{B}^{\prime} \in \mathrm{NC}$ be the noncontextual box for wich the minumum in Eq. (10) is achieved for box $\boldsymbol{B}$, that is, such that $R_{C}(\boldsymbol{B})=$ $S\left(\boldsymbol{B} \| \boldsymbol{B}^{\prime}\right)$. Then, we have

$$
\begin{aligned}
R_{C}\left(\boldsymbol{B}_{\mathrm{f}}\right) & =\min _{\boldsymbol{B}^{*} \in N C} S\left(\boldsymbol{B}_{\mathrm{f}} \| \boldsymbol{B} *\right) \\
& \leq S\left(\boldsymbol{B}_{\mathrm{f}} \| \boldsymbol{B}_{\mathrm{f}}^{\prime}\right) \\
& \leq S\left(\boldsymbol{B} \| \boldsymbol{B}^{\prime}\right) \\
& =R_{C}(\boldsymbol{B}),
\end{aligned}
$$

which concludes the proof.

\section{Appendix E: Proof of Lemma 4}

Eq. (11) is the expression, in our notation, of the $2^{b-1}$ extremal contextual behaviors of the $b$-cycle scenario derived in Theorem 2 of Ref. [31]. In turn, the $2^{b}$ extremal noncontextual behaviors $\boldsymbol{P}_{\mathcal{A} \mid \mathcal{X}}^{(\boldsymbol{\zeta})}$ are expressed [31] as

$$
p_{\mathcal{A} \mid \mathcal{X}}^{(\boldsymbol{\zeta})}(\boldsymbol{a}, \boldsymbol{x})= \begin{cases}1, & \text { if } x_{i}=1 \text { and } a_{2 i-1}=\zeta_{i}+1, \\ 0, & \text { otherwise, }\end{cases}
$$

where $\zeta=\left(\zeta_{1}, \ldots, \zeta_{b}\right)$ is an arbitrary bit string of length $b$ that encodes the deterministic output of each button $i$. Note also that $a_{2 i-1}=\zeta_{i}+1$ is equivalent to $a_{2 i}=\zeta_{i}$. The $2^{b-1}$ behaviors given by Eq. (11), together with the $2^{b}$ behaviors of Eq. (E1), constitute all the extremal points of ND [31]. Hence, any nondisturbing $b$-cycle behavior is a convex combination of $\boldsymbol{P}_{\mathcal{A} \mid \mathcal{X}}^{(\boldsymbol{\zeta})}$ 's and $\boldsymbol{P}_{\mathcal{A} \mid \mathcal{X}}^{(\boldsymbol{\gamma})}$ 's. Thus, we must prove that any particular $\boldsymbol{P}_{\mathcal{A} \mid \mathcal{X}}^{\left(\gamma^{\prime}\right)}$ can be mapped by a wiring in NCW to an arbitrary convex combination of $\boldsymbol{P}_{\mathcal{A} \mid \mathcal{X}}^{(\zeta)}$ 's and $\boldsymbol{P}_{\mathcal{A} \mid \mathcal{X}}^{(\gamma)}$ 's.

First we show that every behavior $\boldsymbol{P}_{\mathcal{A} \mid \mathcal{X}}^{(\gamma)}$ or $\boldsymbol{P}_{\mathcal{A} \mid \mathcal{X}}^{(\xi)}$ can be obtained from any fixed $\boldsymbol{P}_{\mathcal{A} \mid \mathcal{X}}^{\left(\gamma^{\prime}\right)}$ using a wiring in NCW, for arbitrary $\boldsymbol{\gamma}$ and $\boldsymbol{\zeta}$. For $\boldsymbol{P}_{\mathcal{A} \mid \mathcal{X}}^{(\boldsymbol{\gamma})}$ we use as pre-processing the trivial (identity) deterministic box with $b$ input buttons and $b$ output lights, where each input $i$ has only one possible output $i$, and as post-processing the deterministic box $\boldsymbol{B}_{P O S T}^{\gamma^{\prime} \rightarrow \gamma}$ with $2 b$ input buttons and $2 b$ output lights that permutes each $i$-th pair of lights, $2 i-1$ and $2 i$, whenever $\gamma_{i} \neq \gamma^{\prime}{ }_{i}$. For $\boldsymbol{P}_{\mathcal{A} \mid \mathcal{X}}^{(\zeta)}$ we also use as preprocessing the trivial identity box and as post-processing the deterministic box $\boldsymbol{B}_{P O S T}^{\gamma^{\prime} \rightarrow \zeta}$ with $2 b$ input buttons and $2 b$ output lights such that for each $i$-th pair of input (incompatible) buttons, $2 i-1$ and $2 i$, has light $2 i-1+\boldsymbol{\zeta}_{i}$ as deterministic output.

Finally, taking the trivial box as pre-processing and an arbitrary convex combination of the post-processing boxes $\boldsymbol{B}_{P O S T}^{\gamma^{\prime} \rightarrow \gamma}$ and $\boldsymbol{B}_{P O S T}^{\gamma^{\prime} \rightarrow \boldsymbol{\zeta}}$ described above defines a class of wirings in NCW that takes $\boldsymbol{P}_{\mathcal{A} \mid \mathcal{X}}^{\left(\gamma^{\prime}\right)}$ to all convex combinations of the $\boldsymbol{P}_{\mathcal{A} \mid \mathcal{X}}^{(\gamma)}$ 's and $\boldsymbol{P}_{\mathcal{A} \mid \mathcal{X}}^{(\boldsymbol{\zeta})}$ 's, as desired. 